\title{
Orientierung durch Werte
}

\section{Werte als Wegweiser in der lernenden Organisation}

DOI: https://doi.org/10.53349/sv.2021.i2.a84

\begin{abstract}
Eine lernende Organisation zeichnet sich dadurch aus, dass sie sich kontinuierlich weiterentwickelt und Lernen fest in der Organisationskultur verankert ist. Neu erworbenes Wissen sowie die damit verbundenen Erkenntnisse werden im Kollegium weitergegeben und in die schulischen Prozesse integriert. Damit das Kollegium Weiterentwicklung zur Erreichung gemeinsamer Ziele anstrebt, bedarf es einer lernförderlichen Kultur, in der Fehler als Chance zum Lernen verstanden werden. Der folgende Artikel geht der Frage nach, welche Faktoren in der lernenden Schule zur Stärkung einer werteorientierten Schulkultur beitragen.
\end{abstract}

Lernkultur, Dialog, Team-Lernen

„Eine lernende Organisation ist ein Ort, an dem Menschen kontinuierlich entdecken, dass sie ihre Realität selbst erschaffen."

(Senge, 2017, S. 24)

\section{Einleitung}

Unter einer lernenden Organisation wird im schulischen Kontext eine Schule verstanden, die in der Lage ist, neues Wissen zu schaffen, zu erweitern und im Kollegium weiterzugeben sowie dieses Wissen mit den damit verbundenen neuen Erkenntnissen in den Abläufen des Schulalltags anzuwenden. Dass neue Ideen für das Lernen unerlässlich sind, wird zum Selbstverständnis - und zwar unabhängig davon, ob diese aufgrund von Anforderungen, die von extern an eine Organisation herangetragen werden, durch kreative Ideen innerhalb einer Organisation oder aus einer bestimmten Notwendigkeit heraus entstehen. Durch die daraus in weiterer Folge entstehenden organisatorischen Verbesserungen allein wird jedoch noch kei- 
ne lernende Organisation geschaffen. Ohne begleitende Veränderungen hinsichtlich der Art und Weise, wie Prozesse ablaufen und Arbeit erledigt wird, besteht lediglich das Potenzial für Verbesserungen und Entwicklung. In der lernenden Organisation findet Wissen hingegen dauerhaft und nachhaltig Eingang und Anwendung in den Prozessen (Garvin, 1993, S. 80). Begünstigt wird dieser Vorgang durch eine förderliche, unterstützende Kultur, in der die Mitglieder Weiterentwicklung anstreben, die sowohl für das Individuum selbst als auch für das Kollektiv als wertvoll für die Erreichung der gemeinsamen Ziele erachtet wird (Permantier et al., 2021, S. 43).

\section{Auf dem Weg zur lernenden Organisation}

Lernende Organisationen beherrschen nach Garvin (1993) fünf Disziplinen: die systematische Problemlösung, das Experimentieren mit neuen Ansätzen, das Lernen aus der eigenen Erfahrung und der Vergangenheit, das Lernen aus den Erfahrungen und bewährten Verfahren anderer sowie der schnelle, effiziente Wissenstransfer innerhalb der Organisation. Jede dieser Disziplinen geht mit einer bestimmten Denkweise, bestimmten Instrumenten und Verhaltensmustern einher. Viele Organisationen beherrschen diese Aktivitäten zwar bis zu einem gewissen Grad, aber nur wenige sind dauerhaft erfolgreich, weil sie sich in erster Linie auf Problemlösungstechniken konzentrieren und das Lernen und ihr neu erworbenes Wissen nicht dauerhaft, nicht nachhaltig in ihren Prozessen verankern. Dazu braucht es Systeme und Prozesse, welche die kontinuierliche Verbesserung sowie die systematische Suche nach neuen Erkenntnissen unterstützen und dabei helfen, neue Erfahrungen in die Abläufe zu integrieren, damit Lernmanagement effektiv gestaltet werden kann (S. 78ff).

Die Weiterentwicklung einer Schule in eine lernende Organisation stellt einen langfristigen Prozess dar. Einerseits müssen im Sinne von Empowerment die Kompetenzen der Mitarbeiter*innen in den Fokus der Personalarbeit gerückt werden (Fassbender, 1997, S. 60), andererseits dürfen nicht das Können und Wissen des*der Einzelnen im Vordergrund stehen, sondern Kommunikation, Interaktion und Dialog zwischen den Organisationsmitgliedern, wodurch Wissen anderen erst zugänglich gemacht und neues Wissen produziert wird (Güldenberg, 1997, S. 100f).

Durch Dialog wird Team-Lernen unterstützt und individuelle Lernprozesse werden in Gang gesetzt (ebd.). „Das Team-Lernen ist von entscheidender Bedeutung, weil Teams, nicht einzelne Menschen, die elementare Lerneinheit in heutigen Organisationen bilden", betont Senge $(2017$, S. 21) und ergänzt, dass eine Organisation nur dann lernen kann, wenn ihre Teams lernfähig sind. Für einen gelingenden Dialog braucht es das Bewusstsein, dass sich alle Beteiligten als gleichberechtigte und gleichwertige Dialogpartner*innen sehen, die gemeinsam nach neuen Erkenntnissen und neuem Wissen streben. Erfahrene Gesprächspartner*innen, gleichsam „Überwacher*innen“ des Dialogs, stehen beratend und helfend zur Verfügung, solIte der Dialog ins Stocken geraten und in die Diskussion abzugleiten drohen (ebd., S. 267f). 
Das Modell der lernenden Organisation beabsichtigt die Herstellung einer Lernkultur in allen Bereichen (Schlüter, 2007, S. 41f). Über den Reifegrad der Lernkultur und die kulturprägenden Werte einer lernenden Organisation gibt das Leitbild Auskunft (Sonntag, 1997, S. 52f). Der Begriff Leitbild wird im schulischen Bereich oft als Synonym für Vision verwendet, da das Leitbild in vielen Schulen einer Zusammenfassung ihrer Vision entspricht (Buhren \& Rolff, 2016, S. 461). In den nachfolgenden Ausführungen werden die beiden Begriffe Vision und Leitbild daher synonym verwendet, erlauben aber auch ein Verständnis von Leitbild als Vorstufe zur Vision oder umgekehrt.

\title{
Das gemeinsame Leitbild - eine gemeinsame Zukunftsvision
}

\author{
„Wenn es je eine einzelne Führungsidee gab, \\ die Organisationen seit ewigen Zeiten inspiriert hat, \\ so ist es die Fähigkeit, eine gemeinsame Zukunftsvision \\ zu schaffen und aufrechtzuerhalten."
}

(Senge, 2017, S. 19)

Aus dem privatwirtschaftlichen Sektor ist bekannt, dass Unternehmen auf Dauer erfolgreich sind, wenn es eine große Vision, gemeinsame Ziele und Wertvorstellungen gibt. Senge (2017, S. 20) erklärt diese Praxis damit, dass „echte Visionen“ - zum Unterschied von halbherzigen Visionsbekundungen - Mitarbeiter*innen dazu ermuntern, sich aus eigenem Antrieb am Erfolg einer Organisation zu beteiligen. Die Rolle der Führungskraft liegt darin, eine Vision zu entwickeln und diese weiterzutragen, zu vermitteln, die Mitarbeiter*innen anzustiften, die Vision kollektiv umzusetzen. Das bedeutet, dass Führungspersonen als Persönlichkeiten ein plastisches Bild der gemeinsamen Zukunft vermitteln und Zukunftsbilder schaffen können, um echtes, euphorisches Engagement freizusetzen.

Eine erste Konkretisierung der Vision wird im Leitbild niedergeschrieben und formuliert (Dubs, 2005, S. 60). Das Leitbild basiert auf den gelebten Werten, Normen und Zielvorstellungen einer Organisation (Hinterhuber \& Krauthammer, 2015, S. 107) mit dem Ziel zu orientieren und zu motivieren und die eigene Arbeit als sinnstiftend erleben zu lassen (Kadisch, 2017, S. 30). Von Bedeutung ist dabei, dass die Werte, die Stärken und die Potenziale einer Organisation ehrlich und authentisch kommuniziert werden (Permantier et al., 2021, S. 45).

Nach innen bietet das Leitbild den Mitarbeiter*innen und Führungskräften neben der Verschriftlichung von Werten vor allem Orientierung an den langfristigen Perspektiven aus der Vision. Nach außen verdeutlicht es das ethische Selbstverständnis, die Werte der Organisation. Besonders zielführend und sinnstiftend ist ein Leitbild dann, wenn es partizipativ erarbeitet wurde, wenn es emotional berührt, wenn sich alle im Schulgeschehen Beteiligten damit identifizieren können. Als Orientierungshilfe erleichtert das Leitbild die Zielformulierung, als Handlungs- und Entscheidungsgrundlage unterstützt es die Erfüllung von Aufgaben und die Festlegung von Maßnahmen zur Zielerreichung, um die schulische Realität in Richtung des im Leitbild festgeschriebenen Ideals entwickeln zu können (Kapl, 2005, S. 90). Zudem 
wird durch das Niederschreiben und Formulieren von Werten im Leitbild eine Entwicklung innerhalb der Organisation angeregt (Permantier et al., 2021, S. 90).

Durch die konsequente Einbeziehung aller Akteur*innen erfolgt die glaubhafte Umsetzung der Werte im schulischen Handeln; sie führt damit zu einer Stärkung der werteorientierten Schulkultur. Ein top-down verordnetes, von der schulischen Führungskraft definiertes Leitbild ohne Einbindung des Kollegiums führt hingegen meist nicht zum Erfolg, weil die aktive Beteiligung, basierend auf dem Interesse, dem Mitwirken und dem Mitgestalten fehlt, und sich die Mitglieder der Organisation nicht immer mit den vorgegebenen Werten identifizieren können (Kadisch, 2017, S. 30f).

Visionen verändern sich und erfordern eine kontinuierliche Auseinandersetzung mit dem existierenden Leitbild. Hinterhuber und Krauthammer (2015, S. 117) betonen in diesem Zusammenhang, dass ein Leitbild nur dann wirksam ist, „wenn es eine Bewegung in die Zukunft auslöst". Vorhandene Leitbilder, Werteprofile und Führungsgrundsätze sind nicht zu verwerfen, sondern sind in Entwicklungsprozessen stets auf Vereinbarkeit mit neuen Visionen zu überprüfen und behutsam anzupassen (Kadisch, 2019, S. 287), wobei es wirksam ist, wenn sich das Kollegium regelmäßig, beispielsweise im Rahmen von pädagogischen Tagen, gemeinsam über die gelebten, handlungsleitenden Werte austauscht (Permantier et al., 2021, S. 46). Bei allen Veränderungen gilt es zu beachten, dass die Erarbeitung eines bereits bestehenden Leitbildes stets wertgeschätzt werden soll (Kadisch, 2019, S. 287).

Neben der Vision verändern sich auch die Werte, die in der Kommunikation sichtbar werden und im Rahmen der langfristigen Entwicklung der Kultur Orientierung geben (Permantier et al., 2021, S. 46). Werte sind nicht statisch, sondern dynamisch und orientieren sich auch an der gesellschaftlichen Entwicklung (Permantier et al., 2021, S. 95). Im Rahmen des WerteEntwicklungsprozesses wird auch der Bedarf an Veränderung innerhalb der Organisation aufgezeigt (Permantier et al., 2021, S. 83).

Es liegt an der Schulleitungsperson, durch Vermittlung der Vision und das Vorleben von Werten sowie durch authentische Kommunikation dafür zu sorgen, dass die Mitarbeiter*innen Teil dieser Vision sein möchten und die Umsetzung der daraus entstehenden Maßnahmen mitgetragen werden (Kadisch, 2019, S. 279). Für die Akzeptanz der Vision kommt der Steuerung der Kommunikation innerhalb der Schule besondere Bedeutung zu, um ein Selbstverständnis für die Notwendigkeit von Veränderungen und Mitverantwortung im Kollegium zu entwickeln (Fassbender, 1997, S. 60).

So identifizierter Veränderungsbedarf muss als sinnstiftend empfunden und von den gemeinsamen Werten getragen werden - von oben verordnet, führt Veränderung daher nicht zu Engagement. Die für Anregungen offene und kritikfähige Führungskraft fördert hingegen einen hierarchieübergreifenden Austausch, eine bottom-up angetriebene Entwicklung, die auf Stärken und Potenziale fokussiert, wodurch die Veränderungsbereitschaft steigt und Neugier auf Neues geweckt wird (Permantier et al., 2021, S. 54). 


\title{
\# schuleverantworten
}

führungskultur_innovation_autonomie

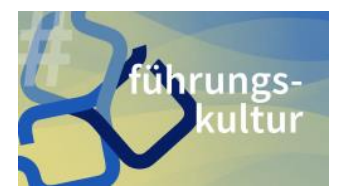

Die Umsetzung einer gemeinsamen Vision gelingt nur, wenn eigene Wege und Fehler zugelassen, zur Selbstmotivation ermutigt und Lernen ermöglicht wird, also eine Auseinandersetzung mit den Werten in der Kultur erfolgt und die Umgestaltung in eine lernende Organisation als Langfristintervention gesehen wird (Fassbender, 1997, S. 63).

\section{Kulturprågende Werte bestimmen Einstellungen}

\author{
„Werte bestimmen Einstellungen. \\ Einstellungen bestimmen Verhalten." \\ (Hinterhuber \& Krauthammer, 2015, S. 96)
}

Eine lernende Organisation zeichnet sich dadurch aus, dass alle Potenziale genutzt werden und Lernen fest in der Organisationskultur verankert ist (Sonntag, 1997, S. 51). Die Lernkultur ist gekennzeichnet durch Reflexionsfähigkeit und flexible Anpassung an Umweltveränderungen (ebd., S. 52). Für eine lernförderliche Organisationskultur in der Schule braucht es die Unterstützung durch die Führungskraft, die offen ist für innovative, interdisziplinäre Lernformen und Herangehensweisen bei der Erprobung neuer Konzepte. Die Schulleitung in der lernenden Organisation fördert Kommunikation und Wissensaustausch, ist aufgeschlossen gegenüber Neuentwicklungen, erkennt Lernen als integralen Bestandteil der strategischen Ausrichtung und beteiligt alle Organisationsmitglieder an Lern- und Veränderungsprozessen (ebd., S. 52f).

Der schulischen Führungskraft kommt in der lernenden Organisation daher eine wesentliche Rolle zu, denn für die erfolgreiche Umsetzung der Lernkultur braucht es eine engagierte, gegenüber Neuentwicklungen aufgeschlossene Führung, die Personalentwicklung und potenzialfördernde Maßnahmen sowie Wissen als integrale Bestandteile in der schulischen Bildungsarbeit erkennt (Sonntag, 1997, S. 53).

Burow (2016, S. 29) hebt den Aufbau einer Kultur der Wertschätzung durch die Förderung von Wohlbefinden als wichtigste Schulleitungsaufgabe hervor. Obwohl Kultur als langfristige Entwicklung zu sehen ist (Permantier et al., 2021, S. 43), kann bereits mit geringem Aufwand in kürzester Zeit Wirkung und damit eine positive Beeinflussung aller schulischen Bereiche erzielt werden (Burow, 2016, S. 29). An der Kultur direkt kann nicht gearbeitet werden, sie baut auf Werten auf und ist das Ergebnis von Kommunikation innerhalb eines Systems (Permantier et al., 2021, S. 50).

Eine von Wertschätzung geprägte Kommunikationskultur basiert vor allem auf der Verbesserung von Kommunikation sowie auf Ver- und Zutrauen, wodurch die Identifikation mit der Organisation und das Vertrauen der Mitarbeiter*innen in ihre eigenen Fähigkeiten und Potenziale gestärkt werden (Kadisch, 2019, S. 276). Eine positive Fehlerkultur und Vertrauen sind die Grundlage für die Entwicklung einer Wertschätzungskultur, in der Erfahrungen gesammelt werden und in der Fehler als Chance zum Lernen begriffen werden (Kadisch, 2017, S. 29). 
Vertrauen und erlebte Wertschätzung fördern Verbundenheit und Identifikation, setzen aber Teilhabe und Partizipation voraus. Daraus entwickelt sich ein Wir-Gefühl und Verantwortungsbereitschaft als Basis dafür, dass gemeinsame Werte gelebt werden (Kadisch, 2019, S. $276,293)$. Veränderungsprozesse, die von den schulischen Akteur*innen aktiv und bewusst initiiert und reflektiert werden, entstehen in weiterer Folge aus dem Blickwinkel der Selbstwahrnehmung und Selbstverantwortung heraus (Kadisch, 2017, S. 29).

Führungskräfte, die wertschätzend und authentisch führen, inspirieren und fördern durch ihre Vorbildwirkung Identifikation und Neugier (Kadisch, 2019, S. 288). Wertschätzung und Vertrauen sind entscheidende Faktoren dafür, dass sich die Akteur*innen am Schulstandort wohlfühlen und dadurch ihr kreatives Potenzial entfalten können (Kadisch, 2017, S. 23). Die schulische Führungskraft unterstützt als Normstifter*in und Vorbild die Etablierung dieser Werte in der Organisationskultur und unterstützt dadurch maßgeblich eine werteorientierte Organisationsentwicklung (ebd., S. 23f).

\section{Fazit}

Im vorliegenden Artikel wird ausgeführt, welche Faktoren zur Stärkung einer werteorientierten Schulkultur in der lernenden Schule beitragen. Dabei sei besonders die Rolle der schulischen Führungskraft bei der Vermittlung der gemeinsamen Zukunftsbilder und beim Vorleben von Werten hervorzuheben. Kulturprägende Werte wie Vertrauen und Wertschätzung sind Voraussetzung für die Schaffung einer positiven Fehlerkultur, damit Fehler nicht als Mangel, sondern als Lernpotenzial und somit als wesentlicher Beitrag für Lernerfolg begriffen werden.

Durch die kontinuierliche Auseinandersetzung mit dem Leitbild werden Werteprofile und Führungsgrundsätze stets auf die Vereinbarkeit mit neuen Visionen überprüft. Dazu braucht es eine für Neuentwicklungen aufgeschlossene Führungskraft, welche die Bedeutung von Kommunikation und Team-Lernen erkennt und in der Personalarbeit gezielt berücksichtigt.

\section{Literaturverzeichnis}

Buhren, C. G., \& Rolff, H.-G. (2016). Personalmanagement. In H. Buchen \& H.-G. Rolff (Hrsg.), Professionswissen Schulleitung (4., überarbeitete und erweiterte Auflage, S. 450-544). Beltz.

Burow, O.-A. (2016). Wertschätzende Schulleitung: Der Weg zu Engagement, Wohlbefinden und Spitzenleistung: wie Schulen zukunftsfähig werden. Beltz.

Dubs, R. (2005). Die Führung einer Schule: Leadership und Management (2. vollst. neu bearb. Aufl). SKV.

Fassbender, P. (1997). Auf dem Weg zum lernenden Unternehmen. In Dr. Wieselhuber und Partner Unternehmensberatung (Hrsg.), Handbuch lernende Organisation: Unternehmens- und Mitarbeiterpotentiale erfolgreich erschließen (S. 55-66). Springer.

Garvin, D. A. (1993). Building a Learning Organization. Harvard Business Review, 71(4), 78-81. 


\section{\# schuleverantworten}

führungskultur_innovation_autonomie

Güldenberg, S. (1997). Wissensmanagement und Wissenscontrolling in lernenden Organisationen: Ein systemtheoretischer Ansatz. Dt. Univ.-Verl.

Hinterhuber, H. H., \& Krauthammer, E. (2015). Leadership - mehr als Management: Was Führungskräfte nicht delegieren dürfen (5. Aufl). Springer Gabler.

Kadisch, I. (2017). Werteorientierte Organisationsentwicklung: „House of Feel Good“: macht Unternehmen stark und menschlich. tredition.

Kadisch, I. (2019). Auf der Suche nach der Seele der Organisation. Wertefundierte Organisationsentwicklung fragt Warum. In I. Kadisch (Hrsg.), Wertefundierte Organisationsentwicklung: Methoden, Ansätze, Wirkfaktoren (S. 275-308). tredition.

Kapl, G. (2005). Leitbildentwicklung - ein schöpferischer Prozess I. In F. Auinger, W. R. Böhnisch, \& H. Stummer (Hrsg.), Unternehmensführung durch Werte: Konzepte, Methoden, Anwendungen (S. 89-106). Dt. Univ.-Verl.

Permantier, M., Bischoff, D., \& Korpas, B. (2021). Werte wirken: Strategie, Marke und Kultur mit Werten entwickeln. Franz Vahlen.

Schlüter, A. (2007). Die „Lernende Organisation“ als mentales Modell für die Personal- und Organisationsentwicklung von Weiterbildungseinrichtungen. In K. Dollhausen \& E. Nuissl von Rein (Hrsg.), Bildungseinrichtungen als „lernende Organisationen“? Befunde aus der Weiterbildung (S. 41-56). Dt. Univ.-Verl.

Senge, P. M. (2017). Die fünfte Disziplin: Kunst und Praxis der lernenden Organisation (11. Auflage). Schäffer-Poeschel.

Sonntag, K. (1997). Wege zur Lernkultur und organisationalen Effizienz. In Dr. Wieselhuber und Partner Unternehmensberatung (Hrsg.), Handbuch lernende Organisation: Unternehmens- und Mitarbeiterpotentiale erfolgreich erschließen (S. 45-54). Springer.

\section{Autorin}

Michaela Tscherne, Prof. Dr. BEd MBA MSc

Professorin und Qualitätsbeauftragte an der Pädagogischen Hochschule Niederösterreich; Arbeits-und Forschungsschwerpunkte: Leadership, Schulautonomie, Personalentwicklung, Berufsorientierung; Publikationen im Bereich Leadership.

Kontakt: michaela.tscherne@ph-noe.ac.at 